\title{
ICHTHYOFAUNA OF SAN IGNACIO LAGOON, BAJA CALIFORNIA SUR, MEXICO
}

\section{ICTIOFAUNA DE LAGUNA SAN IGNACIO, BAJA CALIFORNIA SUR, MEXICO}

\author{
Gustavo D. Danemann 1 \\ José de la Cruz-Agüero² \\ 1 Universidad Autónoma de Baja California Sur \\ Departamento de Biología Marina \\ Apartado postal 19-B \\ La Paz, Baja California Sur, México, C.P. 23081 \\ 2 Centro Interdisciplinario de Ciencias Marinas \\ Departamento de Biología Marina \\ Apartado postal 592 \\ La Paz, Baja California Sur, México, C.P. 23000 \\ Recibido en mayo de 1992; aceptado en abil de 1993
}

\begin{abstract}
Eighty-one species of fishes were recorded from San Ignacio Lagoon, Baja California Sur, Mexico. Data were drawn from analysis of the diet of seven species of fish-eating birds, along with additional survey collections and fish taken by fishermen in the area. This is the first comprehensive check-list of fishes for this area. Five species reported here extend their known distribution range northward.
\end{abstract}

\section{RESUMEN}

Se registraron ochenta y una especies de peces de la Laguna San Ignacio, Baja California Sur, Mćxico. El presentc listado es cl primero que se realiza sobre la ictiofauna de esta zona, y es el resultado del análisis de los hábitos alimentarios de siete especies de aves ictiófagas, recolecciones de prospección y muestras adicionales proporcionadas por los pescadores del área. Cinco de las especies aquí listadas extienden su distribución geográfica conocida hacia el norte.

\section{INTRODUCTION}

Several fish collections have been reported from the western Pacific coast of the Baja California peninsula (e.g. Streets, 1877; Osburn and Nichols, 1916; Wales, 1932). However, no report includes fishes from San Ignacio Lagoon, Baja California Sur. Although Hubbs (1952) based his description of the subspecies Exerpes asper earli (Labrisomidae) on specimens from this lagoon and collected fish at this locality around 1948 (Clark Hubbs, personal communication), a full account of those collections was never published and is not available.

\section{INTRODUCCION}

Existen varios informes sobre recolecciones de peces realizadas en la costa occidental de la Península de Baja California (p. ej. Streets, 1877; Osburn y Nichols, 1916; Wales, 1932). Sin embargo, ninguna publicación incluye peces de Laguna San Ignacio, en Baja California Sur. Aunque Hubbs (1952) basó su descripción de la subespecie Exerpes asper earli (Labrisomidae) en especímenes de esta laguna, donde realizó recolecciones alrededor de 1948 (Clark Hubbs, comunicación personal), no publicó una relación completa y no se dispone de un registro accesible. 
During 1989, Danemann carried out his thesis research at Ballena Island in San Ignacio Lagoon (Danemann, 1991). As part of the study, diet samples from seven species of fish-eating birds were collected. Combined with additional collections made from November 1990 to February 1993 and records of species included in the commercial fishery of the area, this paper is a first, but preliminary, listing of the ichthyofauna of San Ignacio Lagoon. We recorded 81 species of fishes.

\section{STUDY AREA}

San Ignacio Lagoon is located on the western coast of the Baja California peninsula, between $26^{\circ} 38^{\prime}$ and $27^{\circ} 00^{\prime} \mathrm{N}$ and $113^{\circ} 06^{\prime}$ and $113^{\circ} 18^{\prime} \mathrm{W}$ (Fig. 1). It has an area of $175 \mathrm{~km}^{2}$ (Contreras, 1985). Depths average 2 to $4 \mathrm{~m}$ in most of the lagoon, and up to $18 \mathrm{~m}$ in the channels that connect it with the ocean. Large masses of Zostera marina account for most of the aquatic vegetation. The coastline is characterized by sandy beaches, mud flats, mangroves, salt marshes, and a few rocky areas. Low tides expose large shoals that extend into the lagoon. The main water body is the northern arm of the lagoon. A second arm running eastward includes most of the mangroves. Several fishing camps are located along the coastline and an artisanal fishery has been developed.

Ballena Island (also called Whale, Pelícanos, Garza or Zopilotes) lies in the northern section of the lagoon (Fig. 1). It covers $1.3 \mathrm{~km}^{2}$ and, at its closest point, is $1,700 \mathrm{~m}$ from land. Sandy beaches and mud flats constitute most of the island shoreline.

\section{METHODS}

Research on Bailena Island was carried out from January to September 1989. Seven species of fish-eating birds breed there (Danemann, 1991; Danemann and Guzmán, 1992): osprey, Pandion haliaetus (Linnaeus, 1758); brown pelican, Pelecanus occidentalis Linnaeus, 1766; double-crested cormorant, Phalacrocorax auritus (Lesson, 1831); western gull, Laızs occidentalis Audubon, 1839; Caspian tern, Stema caspia Pallas, 1770; reddish egret, Egretta nufescens (Gmelin, 1789) and great blue heron, Ardea herodias Linnaeus, 1758. Although some of these spe-
Durante 1989, se desarrolló un trabajo de tesis en Isla Ballena, Laguna San Ignacio (Danemann, 1991). Como parte de este estudio, se recogieron muestras de la dieta de siete especies de aves ictiófagas. Estos datos se complementaron con recolecciones adicionales realizadas de noviembre de 1990 a febrero de 1993, así como con el registro de las especies incluidas en la pesquería de la zona, que constituye un primer listado de la ictiofauna de Laguna San Ignacio. En total, se registraron ochenta y una especies de peces.

\section{AREA DE ESTUDIO}

Laguna San Ignacio se localiza en la costa occidental de la Península de Baja California, entre los $26^{\circ} 38^{\prime}$ y $27^{\circ} 00^{\prime} \mathrm{N}$ y los $113^{\circ} 06^{\prime}$ y $113^{\circ} 18^{\prime} \mathrm{O}$ (Fig. 1). Tiene un área de $175 \mathrm{~km}^{2}$ (Contreras, 1985). En la mayor parte de la laguna, la profundidad media es de 2 a $4 \mathrm{~m}$, y alcanza $18 \mathrm{~m}$ en los canales que la conectan con el océano. La mayor parte de la vegetación acuática está constituida por Zostera marina. La costa presenta playas de arena, bajos lodosos, manglares, marismas, y unas pocas zonas rocosas. Las mareas bajas dejan al descubierto bajos lodosos amplios que se extienden dentro de la laguna. El brazo norte de la laguna conforma el principal cuerpo de agua. Un segundo brazo se extiende hacia el este e incluye la mayor parte de los manglares. A lo- largo de la costa existen varios campos pesqueros, donde se ha desarrollado una pesquería artesanal.

Isla Ballena (también llamada Pelícanos, Garza o Zopilotes) se localiza en la sección norte de la laguna (Fig. 1). Tiene un área de $1.3 \mathrm{~km}^{2} \mathrm{y}$, en su punto más cercano, está a $1,700 \mathrm{~m}$ de la orilla de la laguna. La costa de la isla está constituida en su mayor parte por playas de arena y lodazales.

\section{METODOS}

La investigación en Isla Ballena se llevó a cabo de enero a septiembre de 1989. En esta localidad anidan siete especies de aves ictiófagas (Danemann, 1991; Danemann y Guzmán, 1992): águila pescadora, Pandion haliaetus (Linnaeus, 1758); pelícano pardo, Pelecanus occidentalis Linnaeus, 1766; cormorán de doble cresta, Phalacrocorax auritus (Lesson, 1831); gaviota occidental, Larus occidentalis 


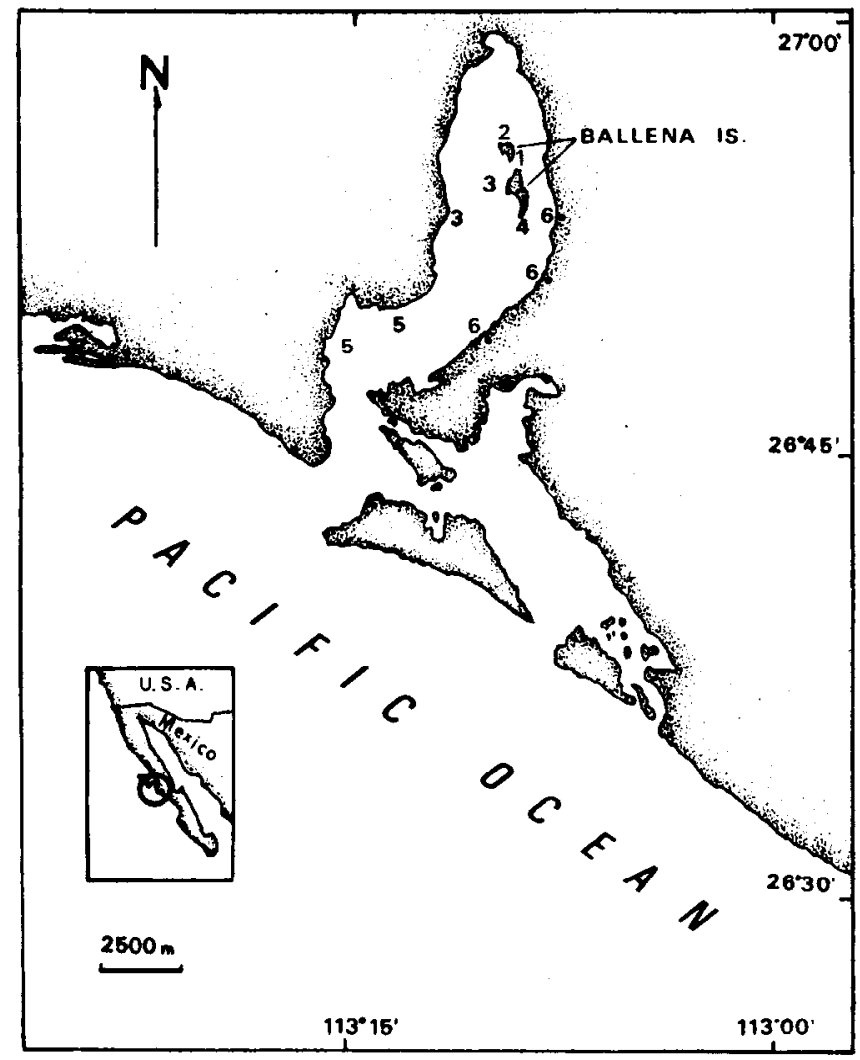

Figure 1. Map of San Ignacio Lagoon, Baja California Sur, Mexico, showing the location of seining and collecting areas. 1) Collecting of fish remains from bird diets (Ballena island). 2) Spearfishing on rocky bottom (north coast of Ballena island). 3) Surface gillnet seining (deep channel). 4) Surface gillnet seining (shallow sandy beach). 5) Trawling area (mud bottom covered with Zostera marina). 6) Fishing camps.

Figura 1. Mapa de Laguna San Ignacio, Baja California Sur, México, en el cual se señala la localización de las áreas de recolección y captura de peces. 1) Recolección de restos de peces provenientes de la dieta de las aves (Isla Ballena). 2) Captura con arpón sobrc fondo rocoso (costa norte de Isla Ballena). 3) Captura con red agallera de superficie (canal profundo). 4) Captura con red agallera de superficie (playa arenosa baja). 5) Zona de arrastres (fondo lodoso cubierto con Zostera marina). 6) Campos pesqueros.

cies may also feed in the open ocean outside the lagoon, all of them were seen fishing in the lagoon and around Ballena Island.

Diet samples were obtained by collecting portions of, or complete fish, dropped in the nest or nesting areas, as well as chick and adult regurgitates. Fresh samples were preserved in $10 \%$ formalin in the field, and were
Audubon, 1839; golondrina marina caspia (Stema caspia Pallas, 1770); garza rojiza, Egretta rufescens (Gmelin, 1789), y garza azul, Ardea herodias Linnaeus, 1758. Si bien es posible que algunas de estas especies se alimenten en el océano, fuera de la laguna, todas ellas fueron vistas alimentándose dentro de la laguna y alrededor de Isla Ballena. 
later stored in $70 \%$ ethyl alcohol. Some samples were kept in their already sun-dried condition.

Additional samples were obtained from fishermen, using gill nets, or by preserving any fish remains found on the island.

Further collections were performed in November 1990, May, August and November 1992, and February 1993. During these visits, trawls and gill nets were used on sandy and nud bottoms in the north inlet of the lagoon, as well as spearguns on the rocky north shore of Ballena Island (Fig. 1). Trawls measured $9 \mathrm{~m}$ long, with an opening of $8 \mathrm{~m}$ and a mesh size of $3 \mathrm{~cm}$. Trawls were used at $4 \mathrm{~m}$ depth during 15 minutes each time. Gill nets measured $140 \mathrm{~m}$ long and $3 \mathrm{~m}$ high, with mesh sizes of 9 and $0.5 \mathrm{~cm}$.

All samples were identified and measurcd. Specimens of each one of the species listed are kept in the Ichthyological Collection of the Centro Interdisciplinario de Ciencias Marinas (CICIMAR-IPN collection numbers 2310-2363, 2379:1-608 and 2764-2792), in La Paz, Baja California Sur, Mexico.

\section{RESULTS}

We identified 81 species (seven to genus or doubtful species only), belonging to 72 genera, 48 families and 19 orders (classification of Eschmeyer, 1990) (table 1). The diet of the seven fish-eating birds included 33 of the species recorded. Smallest prey was Ilypuus gilberti (Eigenmann and Eigenmann, 1889), $28 \mathrm{~mm}$ total length, taken by a reddish egret. Largest prey was Cynoscion parvipinnis Ayres, $1861,580 \mathrm{~mm}$ total length, taken by an osprey. The size of fish collected with fishing gear ranged from $30 \mathrm{~mm}$, Eucinostomus dowii (Gill, 1863), to $1,800 \mathrm{~mm}$. Sphyma zygaena Linnaeus, 1758.

\section{DISCUSSION}

Although some of the specimens collected were not in very good condition, identification was usually possible.

The anchovies Anchovia magdalenae Hildebrand, 1943 and Anchoa lucida (Jordan and Gilbert, 1882) have not been previously recorded north of Magdalena Bay (about $24030^{\prime} \mathrm{N}$; Whitehead et al., 1988). The haemulids Orthopristis reddingi Jordan and
Las muestras de la dieta fueron obtenidas recogiendo peces o porciones de peces dejados por las aves en los nidos o áreas de anidación, así como regurgitados de pollos y adultos. Estas muestras fueron fijadas en el campo con formol al $10 \%$, y se preservaron posteriormente en alcohol etílico al $70 \%$. Algunas de ellas se hallaron deshidratadas por el sol y se conservaron en ese estado.

Se obtuvieron muestras adicionales a través de los pescadores del área, utilizando redes agalleras, y preservando cualquier resto de pez hallado en la isla.

Luego del estudio realizado en 1989 , se realizaron recoleccionesen noviembre de 1990 , mayo, agosto y noviembre de 1992, y febrero de 1993. En estas oportunidades se utilizaron redes de arrastre $y$ agalleras en fondos arenosos y lodosos en el brazo norte de la laguna, así como arpones en la costa rocosa del norte de Isla Ballena (Fig. 1). Se usaron redes de arrastre de $9 \mathrm{~m}$ de longitud, con una abertura de $8 \mathrm{~m}$ y luz de malla de $3 \mathrm{~cm}$. Los lances se realizaron a $4 \mathrm{~m}$ de profundidad, con una duración de 15 minutos por arrastre. Las redes agalleras utilizadas midieron $140 \mathrm{~m}$ de longitud, $3 \mathrm{~m}$ de caída, y tuvieron luces de malla de 9 y $0.5 \mathrm{~cm}$.

Todas las muestras fueron identificadas y medidas. Se conservan en la Colección Ictiológica del Centro Interdisciplinario de Ciencias Marinas (CICIMAR-IPN, La Paz, Baja California Sur), bajo los números 23102363, 2379: 1-608, y 2764-2792).

\section{RESULTADOS}

Se identificaron 81 especies (siete de ellas sólo hasta género o especie dudosa), pertenecientes a 72 géneros, 48 familias y 19 órdenes (clasificación de Eschmeyer, 1990) (tabla 1). La dieta de las aves estudiadas incluyó 33 de las especies registradas. La presa más pequeña fue Ilypnus gilberti (Eigenmann y Eigenmann, 1889), con una longitud total de $28 \mathrm{~mm}$, capturada por una garza rojiza. La presa más grande fue Cynoscion parvipinnis Ayres, 1861, con una longitud total de $580 \mathrm{~mm}$, capturada por un águila pescadora. La talla de los peces recolectados con artes de pesca presentó un intervalo de $30 \mathrm{~mm}$ (Eucinostomus dowii [Gill, 1863]) a 1,800 mm (Sphyma zygaena Linnaeus, 1758). 
Danemann y De la Cruz-Agüero: Icthyofauna of San Ignacio Lagoon

Table 1. Species collected in San Ignacio Lagoon, Baja California Sur, Mexico. $\mathbf{N}=$ northern (temperate) species; $S=$ southern (tropical) species; $W=$ wide range in Eastern Pacific; ${ }^{*}=$ new northerly record. Classification following Eschmeyer (1990). See text for explanation of criteria for species separation.

Tabla 1. Especies recolectadas en Laguna San Ignacio, Baja California Sur, México. $\mathbf{N}=$ especie norteña (templada); $\mathbf{S}=$ especie sureña (tropical); $\mathbf{W}=$ distribución amplia en el Pacífico oriental; * = extensión de la distribución hacia el norte. Clasificación según Eschmeyer (1990). Ver texto para la explicación de los criterios utilizados en la separación de las especies.

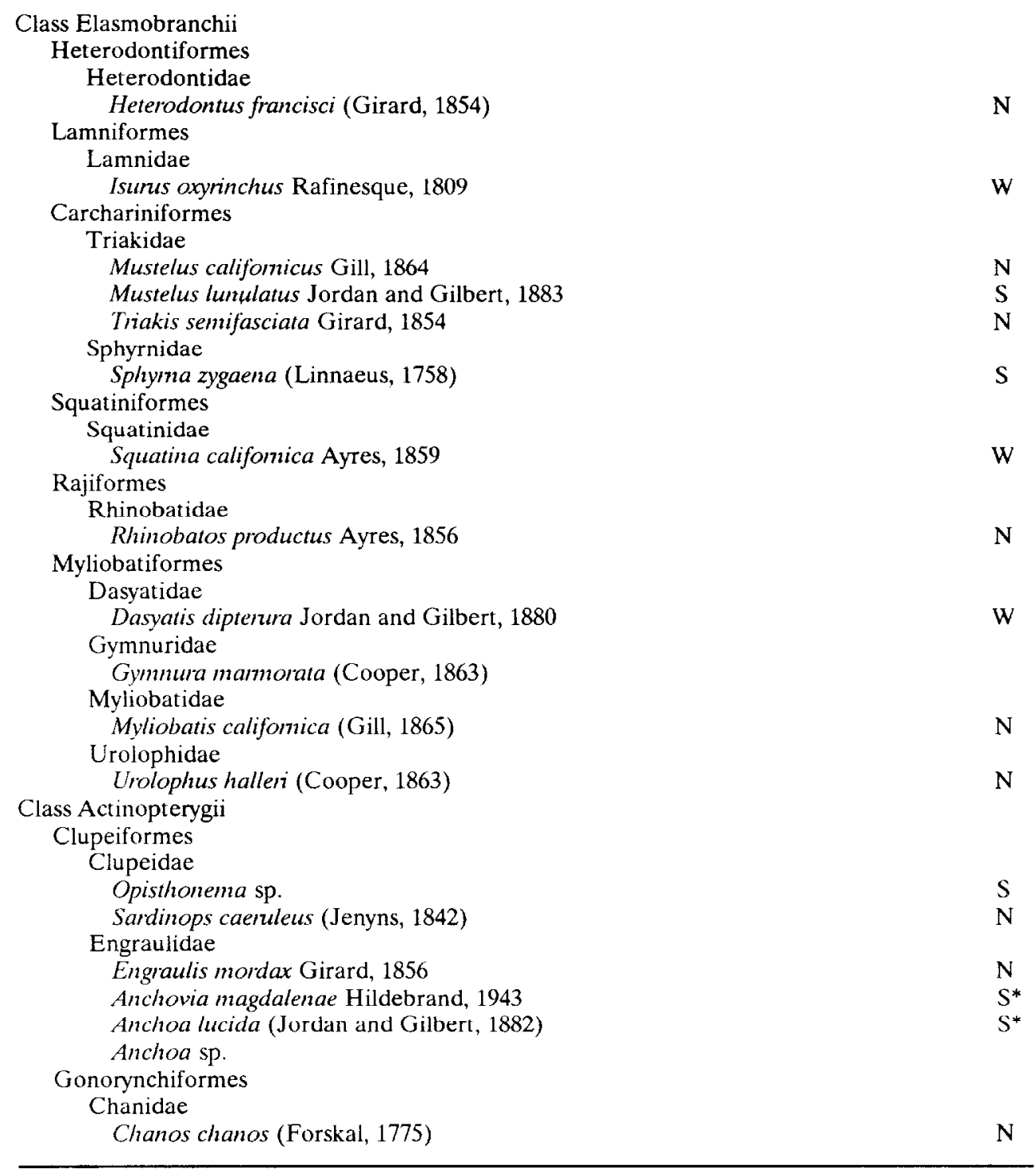


Table 1 (Cont.)

Siluriformes

Ariidac

Bagre panamensis (Gill, 1863)

Arius sp.

Aulopifonnes

Synodontidae

Synodus lucioceps (Ayres, 1855) N N

Ophidiiformes

Ophidiidae

Ophidion scrippsae Hubbs, $1916 \quad$ N

Atheriniformes

Atherinidae

$\begin{array}{ll}\text { Atherinopsis califomiensis Girard, } 1854 & \mathrm{~N}\end{array}$

Atherinops affinis (Ayres, 1860)

$\mathrm{N}$

Cyprinodontiformes

Cyprinodontidae

Fundulus parvipinnis Girard, $1854 \quad$ N

Beloniformes

Belonidae

Strongylura exilis (Girard, 1854) S S

Hemiramphidae

Hyportiamphus unifasciatus (Ranzani, 1842) S

Syngnathiformes

Syngnathidae

Syngnathus leptorhynchus Girard, 1854

Hippocampus ingens Girard, 1858

$\mathrm{N}$

S

Scorpaeniformes

Scorpaenidae

Scorpaena guttata (Girard, 1854) N N

Perciformes

Serranidae

Epinephelus analogus Gill, 1864

$S$

Paralabrax nebulifer Girard, 1854

$\mathrm{N}$

Paralabrax maculatofasciatus (Steindachner, 1868) N N

Carangidae

Oligoplites altus (Gunther, 1868)

Trachinotus shodopus Gill, 1863

$S$

Trachinotus paitensis Cuvier, 1832

$S$

Seriola dorsalis (Gill, 1863)

$S$

Gerreidae

Eucinostomus dowi (Gill, 1863)

Eucinostomus sp.

Haemulidae

Xenistius califomiensis (Steindachner, 1875)

$\mathrm{S}$

Anisotremus davidsoni (Steindachner, 1875)

Orthopistis redding Jordan and Richardson, 1895

W

Orthopristis chalceus (Günther, 1864)

$\mathbf{N}$

$N$ $\mathbf{N}$ 
Table 1 (Cont.)

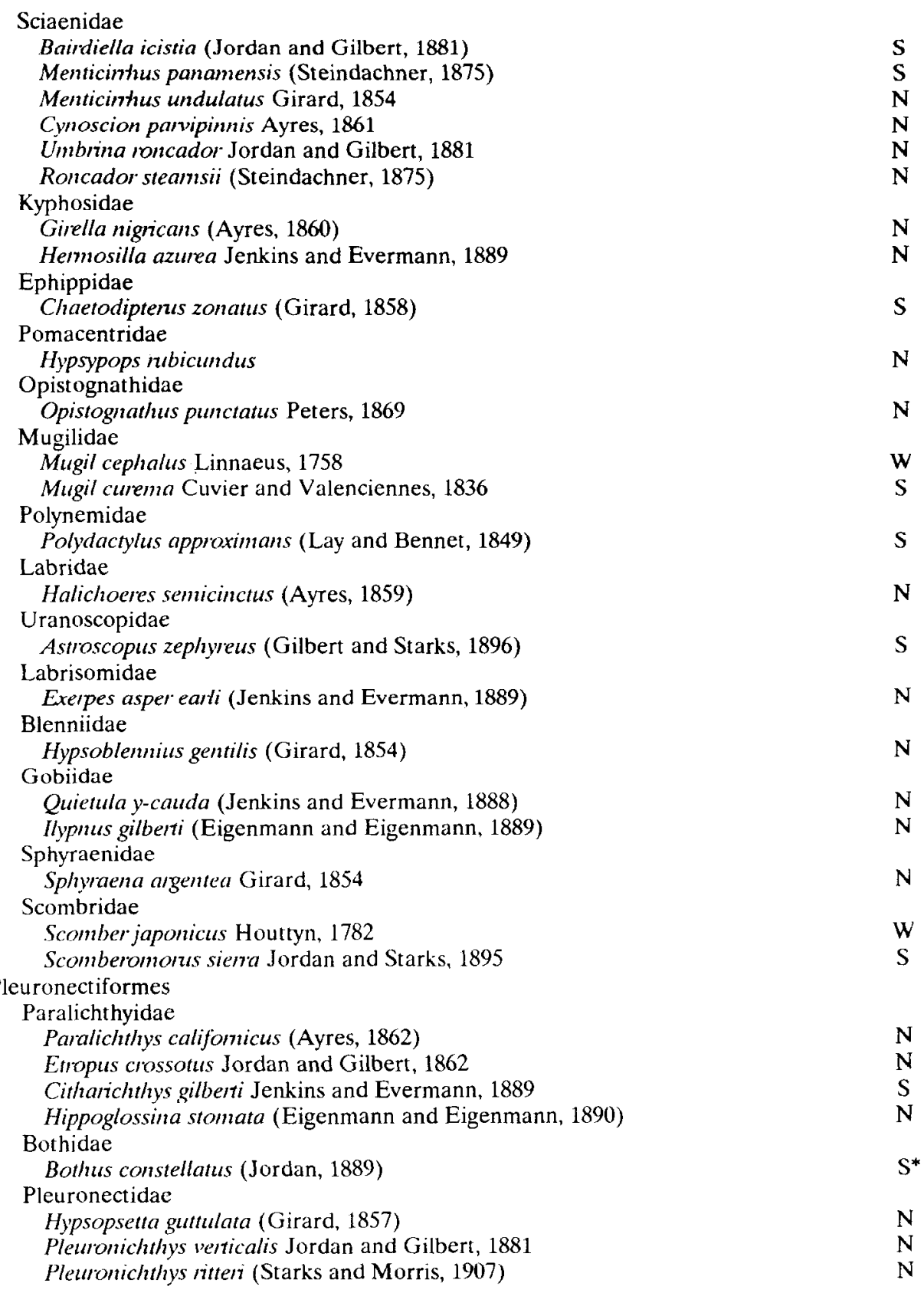


Table 1 (Cont.)

$\begin{array}{lc}\text { Soleidae } & \text { S } \\ \quad \text { Achinus mazatlanus (Steidachner, 1869) } & \text { W } \\ \text { Tetraodontiformes } & \\ \text { Balistidae } & \mathrm{S} \\ \text { Balistes polylepis (Steindachner, 1876) } & \mathrm{S} \\ \text { Tetraodontidae } & \\ \quad \text { Sphoeroides annulatus (Jenyns, 1843) } & \mathrm{S} \\ \text { Sphoeroides sp. } & \\ \text { Diodontidae } & \\ \quad \text { Diodon hystrix Linnaeus, 1758. } & \end{array}$

Richardson, 1895, and $O$. chalceus (Günther, 1864), and the bothid Bothus constellatus (Jordan, 1889) have been reported only to Cabo San Lucas (about $23^{\circ} \mathrm{N}$; Castro et al., 1970; Hensley, in press). Thus, we extend the distribution of these five species north.

Much has been speculated about the boundary between the Eastern Pacific and the California zoogeographical regions (Briggs, 1974). In opposing the idea of a single boundary at Magdalena Bay (to $25^{\circ} \mathrm{N}$ ), Hubbs (1960) pointed out that along the western coast of Baja California, shallow warm-water inlets alternate with cold upwelling areas to create a highly complex intermediate faunal area. In our list, 39 northern species (marked $\mathrm{N}$ in table 1) reach southward to or beyond San Ignacio Lagoon, but not to the Equator. Conversely, 31 southern species (marked $S$ in table 1) reach from south of the Equator to or beyond San Ignacio Lagoon. This apparent mixing of southern (tropical) species and northern (temperate) species in San Ignacio Lagoon shows that a clear boundary does not exist and that, according to Hubbs (1960), not all the northern and southern elements alternate between warm inlets and cool upwelling areas.

\section{ACKNOWLEDGEMENTS}

Danemann's research was supported in part by the Seabird Program coordinated by Juan Guzmán Poo at the Universidad Autónoma de Baja Califonia Sur. Danemann also gratefully acknowledges grants from Sigma $\mathbf{X i}$, the International Osprey Foundation and John Storrer. The 1990-1993 field work was supported by the Ichthyology Program coordinated by Felipe Galván Magaña at the Centro Interdisciplinario de Ciencias Marinas, Instituto Politécnico Na-

\section{DISCUSION}

Aunque algunos de los especímenes recolectados no se encontraban en buenas condiciones, la identificación fue usualmente posible.

Las anchovetas Anchovia magdalenae Hildebrand, 1943 y Anchoa lucida (Jordan y Gilbert, 1882) no habian sido registradas previamente al norte de Bahía Magdalena (alrededor de los 24030', Whitehead et al, 1988). Los haemúlidos Orthopristis reddingi Jordan y Richardson, 1895 y $O$. chalceus (Günther, 1864), así como el bótido Bothus constellatus (Jordan, 1889) se habian reportado sólo hasta Cabo San Lucas (alrededor de los 230N; Castro et al., 1970; Hensley, in press). Consecuentemente, la distribución conocida de estas cinco especies se extiende hacia el norte.

Se ha especulado mucho acerca del límite entre las regiones zoogeográficas del Pacífico oriental y California (Briggs, 1974). En contraposición a la idea de una única frontera en Bahía Magdalena $\left(25^{\circ} \mathrm{N}\right)$, Hubbs (1960) señaló que a lo largo de la costa occidental de Baja California se alternan cuerpos de agua cálida con zonas de surgencias frias, creando un área faunística intermedia y compleja. En nuestra lista, 39 especies norteñas (señaladas con $\mathrm{N}$ en la tabla 1) extienden su distribución al sur hasta Laguna San Ignacio o más allá de ella, sin llegar al Ecuador. Inversamente, 31 especies sureñas (señaladas con $S$ en la tabla 1) extienden su distribución desde el sur del Ecuador hasta Laguna San Ignacio o más allá de ella. Esta aparente mezcla de especies sureñas (tropicales) y norteñas (templadas) en Laguna San Ignacio demuestra que no existe una frontera definida y que, de acuerdo con lo propuesto por Hubbs (1960), no todos los elementos 
cional (CICIMAR-IPN). Francisco Mayoral González, from San Ignacio Lagoon, and personnel from the Ichthyology Program of CICIMAR provided critical help during the study. Peter Whitehead offered useful editorial advice as well as authoritative identification of Clupeiformes.

\section{REFERENCES}

Briggs, J.C. (1974). Marine zoogeography. McGraw-Hill, New York, 475 pp.

Castro A., J.L., Arvizu M., J. y Páez B., J. (1970). Contribución al conocimiento de los peces del Golfo de California. Rev. Soc. Mex. Hist. Nat., 31: 107-181.

Contreras, F. (1985). Las lagunas costeras mexicanas. Centro de Ecodesarrollo, Secretaría de Pesca, México, D.F., 265 pp.

Danemann, G.D. (1991). Amplitud y sobreposición de nichos ecológicos de aves ictiófagas anidantes en Isla Ballena, Laguna San Ignacio, Baja California Sur. Tesis de licenciatura, UABCS, México, $92 \mathrm{pp}$.

Danemann, G.D. and Guzmán, J. (1992). Notes on the birds of San Ignacio Lagoon, Baja California Sur, Mexico. Western Birds, 23: 11-19.

Eschmeyer, W.N. (1990). Catalog of the genera of recent fishes. Publication of the California Academy of Sciences, San Francisco, $697 \mathrm{pp}$.

Hensley, C. Bothidae. In: F. Krupp (ed.), FAO species identification sheets for fishery purpose Eastern Central Pacific. Fishing area 77 . FAO, Rome, Italy (in press).

Hubbs, C.L. (1952). A contribution to the classification of the blennioid fishes of the family Clinidae, with a partial revision of the eastern Pacific forms. Stanford Ichthyol. Bull., 4(2): 41-165.

Hubbs, C. (1960). The marine vertebrates of the outer coast. Symposium: the Biogeography of Baja California and Adjacent Seas. Syst. Zool., 9(3-4): 134-147. norteños y sureños se alternan entre cuerpos de agua cálida y áreas de surgencia frías.

\section{AGRADECIMIENTOS}

La investigación de Danemann fue financiada parcialmente por el Programa de Aves Marinas coordinado por Juan Guzmán Poo en la Universidad Autónoma de Baja California Sur. Además, se recibieron subsidios de Sigma Xi, The International Osprey Foundation y John Storrer. El trabajo de campo de 1990 a 1993 fue financiado por el Programa de Ictiología coordinado por Felipe Galván Magaña en el Centro Interdisciplinario de Ciencias Marinas, Instituto Politécnico Nacional (CICIMAR-IPN). Francisco Mayoral González y el personal del Programa de Ictiología del CICIMAR aportaron una ayuda importante durante el estudio. Peter Whitehead ofreció asesoría editorial e identificó los Clupeiformes recolectados.

Traducido al español por los autores.

Osburn, R.C. and Nichols, J.T. (1916). Shore fishes collected by the "Albatross" expedition in lower California with descriptions of new species. Bull. Amer. Mus. Nat. Hist., 35: 139-181.

Streets, T.H. (1877). Contributions to the natural history of the Hawaiian and Fanning Islands and Lower California, made in connection with the United States North Pacific Surveying Expedition, 1873-75. Ichthyology, I. Fishes of the Upper and Lower California. Bull. U.S. Nat. Mus., 7: 43-56

Wales, J.M. (1932). Reports on two collections of Lower California marine fishes. Copeia, (2): 163-168.

Whitehead, P.J.P., Nelson, G.J. and Wongratana, T. (1988). FAO Species Catalogue, Vol. 7. Clupeoid Fishes of the World (Suborder Clupeoidei). An annotated and illustrated catalogue of the herrings, sardines, pilchards, sprats, anchovies and wolf herrings. Part 2. Engraulididae. FAO Fish. Synop., (125) 7(2): 305-579. 